\title{
SINGULAR SECOND-ORDER MULTIPOINT DYNAMIC BOUNDARY VALUE PROBLEMS WITH MIXED DERIVATIVES
}

\author{
MARTIN BOHNER AND HUA LUO
}

Received 12 September 2005; Accepted 26 October 2005

We study a certain singular second-order $m$-point boundary value problem on a time scale and establish the existence of a solution. The proof of our main result is based upon the Leray-Schauder continuation theorem.

Copyright (c) 2006 M. Bohner and H. Luo. This is an open access article distributed under the Creative Commons Attribution License, which permits unrestricted use, distribution, and reproduction in any medium, provided the original work is properly cited.

\section{Introduction}

Singular nonlinear boundary value problems for differential equations and difference equations have been extensively studied in the literature; see $[1,4,11,12,16,18-22]$ and the references therein. However, the research for singular boundary value problems on time scales is still in its beginning stages. In [8], the authors investigate the existence of a positive solution for the three-point dynamic boundary value problem

$$
y^{\Delta \Delta}+f(x, y)=0, \quad x \in(0,1], y(0)=0, y(p)=y\left(\sigma^{2}(1)\right),
$$

where $\mathbb{T}$ is a time scale, the interval $(0,1] \cap \mathbb{T}$ is abbreviated by $(0,1], p \in(0,1)$ is fixed, and $f(x, y)$ is singular at $y=0$ and possibly at $x=0, y=\infty$.

Throughout we denote by $\mathbb{T}$ a time scale, that is, a nonempty closed subset of the real numbers. In this paper we study the singular second-order $m$-point dynamic boundary value problem

$$
\begin{gathered}
x^{\Delta \nabla}=f\left(t, x, x^{\Delta}\right)+e(t), \quad t \in(a, b], \\
x^{\Delta}(a)=0, \quad x(\sigma(b))=\sum_{i=1}^{m-2} a_{i} x\left(\xi_{i}\right),
\end{gathered}
$$

where $a_{i} \in \mathbb{R}, \xi_{i} \in(a, \sigma(b)), i \in\{1,2, \ldots, m-2\}$, and $f:(a, \sigma(b)) \times \mathbb{R}^{2} \rightarrow \mathbb{R}$ satisfies the Carathéodory conditions, that is, for each $(x, y) \in \mathbb{R}^{2}$, the function $f(\cdot, x, y)$ is measurable 
on $(a, \sigma(b))$ and for (see Definition 2.1) $\nabla$-a.e. $t \in(a, \sigma(b))$, the function $f(t, \cdot, \cdot)$ is continuous on $\mathbb{R}^{2}$. Here we allow $f$ and $e$ to be singular at $t=\sigma(b)$.

In particular, when the nonlinearity $f$ does not contain $x^{\Delta}$, the problem (1.2) has been investigated for the nonsingular case by some authors, see $\mathrm{He}[10]$; when $\mathbb{T}=\mathbb{R}$, the problem (1.2) has been studied for the nonsingular case by Gupta et al. [9] and Ma [14] to name a few. Recently, Ma and O'Regan [16] established the existence of a solution to the singular problem (1.2) in the special case $\mathbb{T}=\mathbb{R}$ by making use of the ideas of $[4,9]$. The motivation for this paper is [16].

The paper is organized as follows. In Section 2, we state some preliminary definitions and results about Lebesgue delta and nabla integrals. We then give all spaces relevant to our work and present the main assumptions ensuring us to obtain the main results. Section 3 is devoted to the study of the properties of Green's function. We also state and prove some lemmas which are required for discussing the problem (1.2). Then we establish the existence of one solution to the problem (1.2) in Section 4.

The time scale related notations adopted in this paper can be found, if not explained specifically, in almost all literature related to time scales. The readers who are unfamiliar with this area can consult for example $[2,3,5-8,10,13,15]$ for details.

\section{The Lebesgue delta and nabla integrals}

The integrals mentioned in this paper refer to the Lebesgue integrals on the time scale $\mathbb{T}$. For the main notions and facts from Lebesgue measures and Lebesgue integrals theory, we refer the reader to [5] and [7, pages 157-163]. Here we give some definitions and lemmas for the convenience of the reader.

Let $\mu_{\Delta}$ and $\mu_{\nabla}$ be the Lebesgue $\Delta$-measure and the Lebesgue $\nabla$-measure on $\mathbb{T}$, respectively. If $A \subset \mathbb{T}$ satisfies $\mu_{\Delta}(A)=\mu_{\nabla}(A)$, then we call $A$ measurable on $\mathbb{T}$ and denote by $\mu(A)$ this same value, named the Lebesgue measure of $A$.

Definition 2.1. Let $P$ denote a proposition with respect to $t \in \mathbb{T}, A \subset \mathbb{T}$.

(1) If there exists $E_{1} \subset A$ with $\mu_{\Delta}\left(E_{1}\right)=0$ such that $P$ holds on $A \backslash E_{1}$, then $P$ is said to hold $\Delta$-a.e. on $A$.

(2) If there exists $E_{2} \subset A$ with $\mu_{\nabla}\left(E_{2}\right)=0$ such that $P$ holds on $A \backslash E_{2}$, then $P$ is said to hold $\nabla$-a.e. on $A$.

(3) If there exists $E_{1} \subset A$ with $\mu_{\Delta}\left(E_{1}\right)=0$ and $E_{2} \subset A$ with $\mu_{\nabla}\left(E_{2}\right)=0$ such that $P$ holds on $A \backslash\left(E_{1} \cup E_{2}\right)$, then $P$ is said to hold $\Delta \nabla$-a.e. on $A$ (or $\nabla \Delta$-a.e. on $A$ ).

(4) If there exists $E \subset A$ with $\mu(E)=0$ such that $P$ holds on $A \backslash E$, then $P$ is said to hold a.e. on $A$.

Clearly, if $P$ holds a.e. on $A \subset \mathbb{T}$, then $P$ holds $\Delta$-a.e. on $A, \nabla$-a.e. on $A$, and $\Delta \nabla$-a.e. on $A$ simultaneously.

Remark 2.2. In the case $\mathbb{T}=\mathbb{R}$, all concepts defined above coincide with that of a.e. on $\mathbb{R}$. In this case we have $\mu_{\Delta}=\mu_{\nabla}=\mu=m$, where $m$ is the usual Lebesgue measure on $\mathbb{R}$. In the case $\mathbb{T}=\mathbb{Z}$, for any subset $E \subset \mathbb{Z}$, we know that $\mu_{\Delta}(E)=\mu_{\nabla}(E)$ coincides with the number of points of the set $E$. So $\mu(E)=\mu_{\Delta}(E)=\mu_{\nabla}(E)=0$ if and only if $E=\varnothing$. 
Combining [7, Theorems 5.82 and 5.84], we have the following example as a further illustration of Definition 2.1.

Example 2.3. Let $f$ be a bounded function defined on the finite closed interval $[r, s]$. Assume that $f$ is regulated. Consider the conditions:

(1) $f$ is Riemann $\Delta$-integrable from $r$ to $s$;

(2) $f$ is Riemann $\nabla$-integrable from $r$ to $s$.

We have

(a) if (1) holds, then $f$ is rd-continuous $\Delta$-a.e. on $[r, s)$;

(b) if (2) holds, then $f$ is ld-continuous $\nabla$-a.e. on $(r, s]$;

(c) if both (1) and (2) hold, then $f$ is continuous $\Delta \nabla$-a.e. on $(r, s)$. If, moreover, $r=$ $\min \mathbb{T}$ and $s=\max \mathbb{T}$, then $f$ is continuous $\Delta \nabla$-a.e. on $[r, s]$. Here the continuity of $f$ at $r$ and $s$ is understood as continuous from the right and left, respectively.

Definition 2.4. For a set $E \subset \mathbb{T}$ and a function $f: E \rightarrow \mathbb{R}$, the Lebesgue integrals of $f$ over E denoted by

$$
\int_{E} f(t) \Delta t, \quad \int_{E} f(t) \nabla t
$$

are called the Lebesgue $\Delta$-integral of $f$ over $E$ and the Lebesgue $\nabla$-integral of $f$ over $E$ on $\mathbb{T}$, respectively. Furthermore, we call $f$ Lebesgue $\Delta$-integrable on $E$ and Lebesgue $\nabla$-integrable on $E$ if $\int_{E} f(t) \Delta t$ and $\int_{E} f(t) \nabla t$ are finite, respectively.

Let $r, s \in \mathbb{T}, r \leq s$. We will use the notations

$$
\int_{r}^{s} f(t) \Delta t=\int_{[r, s)} f(t) \Delta t, \quad \int_{r}^{s} f(t) \nabla t=\int_{(r, s]} f(t) \nabla t,
$$

respectively. Both intervals $[r, r)$ and $(s, s]$ are understood as the empty set.

From [7, page 159], we have that all theorems of the general Lebesgue integration theory hold also for the Lebesgue delta and nabla integrals on $\mathbb{T}$.

Lemma 2.5. If $f$ is Lebesgue $\Delta$-integrable on $[r, s)$, then the indefinite integral $\int_{r}^{t} f(\ell) \Delta \ell$ is absolutely continuous on $[r, s]$.

Lemma 2.6. If $f$ is Lebesgue $\nabla$-integrable on $(r, s]$, then the indefinite integral $\int_{r}^{t} f(\ell) \nabla \ell$ is absolutely continuous on $[r, s]$.

Lemma 2.7. If $f$ is Lebesgue $\Delta$-integrable on $[r, s)$, then $F$ defined by

$$
F(t)=\int_{r}^{t} f(\ell) \Delta \ell, \quad t \in[r, s) \text { satisfies } F^{\Delta}=f \Delta \text {-a.e. on }[r, s) .
$$

Lemma 2.8. If $f$ is Lebesgue $\nabla$-integrable on $(r, s]$, then $F$ defined by

$$
F(t)=\int_{r}^{t} f(\ell) \nabla \ell, \quad t \in(r, s] \text { satisfies } F^{\nabla}=f \nabla \text {-a.e. on }(r, s] .
$$


4 Singular multipoint dynamic boundary value problems

Lemмa 2.9. If $f$ is everywhere finite and absolutely continuous on $[r, s]$, then $f^{\Delta}$ exists $\Delta$-a.e. and is Lebesgue $\Delta$-integrable on $[r, s)$ and satisfies

$$
f(t)=\int_{r}^{t} f^{\Delta}(\ell) \Delta \ell+f(r), \quad t \in[r, s] .
$$

LEMMA 2.10. If $f$ is everywhere finite and absolutely continuous on $[r, s]$, then $f^{\nabla}$ exists $\nabla$-a.e. and is Lebesgue $\nabla$-integrable on $(r, s]$ and satisfies

$$
f(t)=\int_{r}^{t} f^{\nabla}(\ell) \nabla \ell+f(r), \quad t \in[r, s]
$$

LeMma 2.11. Let $f$ be defined on $[r, s]$.

(i) If $f$ is continuous on $[r, s)$, then $\int_{r}^{s} f(\rho(t)) \nabla t=\int_{r}^{s} f(t) \Delta t$;

(ii) if $f$ is continuous on $(r, s]$, then $\int_{r}^{s} f(\sigma(t)) \Delta t=\int_{r}^{s} f(t) \nabla t$.

Proof. We only show (i) as the proof of (ii) is similar to the proof of (i). Since $f$ is continuous on $[r, s)$, there exists $F:[r, s] \rightarrow \mathbb{R}$ such that $F^{\Delta}=f$ holds on $[r, s)$. Then

$$
F^{\nabla}(t)=F^{\Delta}(\rho(t))=f(\rho(t)), \quad \forall t \in(r, s]
$$

by [6, Theorem 8.49$]$. So

$$
\begin{gathered}
\int_{r}^{s} f(t) \Delta t=\int_{r}^{s} F^{\Delta}(t) \Delta t=F(s)-F(r), \\
\int_{r}^{s} f(\rho(t)) \nabla t=\int_{r}^{s} F^{\Delta}(\rho(t)) \nabla t=\int_{r}^{s} F^{\nabla}(t) \nabla t=F(s)-F(r) .
\end{gathered}
$$

This implies that (i) holds.

Now we define the Banach spaces $C[a, \sigma(b)], C^{\Delta}[a, \sigma(b)]$, and $L^{\nabla}(a, \sigma(b)]$ to be the sets of all continuous functions on $[a, \sigma(b)]$ with the sup norm $\|\cdot\|_{\infty}$, all $\Delta$-differentiable functions with continuous $\Delta$-derivative on $[a, \sigma(b)]$ with the norm $\|x\|=\max \left\{\|x\|_{\infty}\right.$, $\left.\left\|x^{\Delta}\right\|_{\infty}\right\}$, and all Lebesgue $\nabla$-integrable functions on $(a, \sigma(b)]$ with the norm $\|x\|=$ $\int_{a}^{\sigma(b)}|x(t)| \nabla t$, respectively. Let

$$
L_{\mathrm{loc}}^{\nabla}(a, \sigma(b))=\left\{x:\left.x\right|_{(a, d]} \in L^{\nabla}(a, d] \text { for every interval }(a, d] \subseteq(a, \sigma(b))\right\} .
$$

We denote by $A C[a, \sigma(b)]$ the space of all absolutely continuous functions on $[a, \sigma(b)]$ and set

$$
A C_{\mathrm{loc}}[a, \sigma(b))=\left\{x:\left.x\right|_{[a, d]} \in A C[a, d] \text { for every interval }[a, d] \subseteq[a, \sigma(b))\right\} .
$$

Let $E$ be the Banach space

$$
E=\left\{x \in L_{\mathrm{loc}}^{\nabla}(a, \sigma(b)):[\sigma(b)-\rho] x \in L^{\nabla}(a, \sigma(b)]\right\}
$$


equipped with the norm

$$
\|x\|_{E}=\int_{a}^{\sigma(b)}[\sigma(b)-\rho(t)]|x(t)| \nabla t
$$

and let $X$ be the Banach space

$$
X=\left\{u \in C^{\Delta}[a, \sigma(b)): u \in C[a, \sigma(b)], \lim _{t \rightarrow \sigma(b)}[\sigma(b)-t] u^{\Delta}(t) \text { exists }\right\}
$$

equipped with the norm

$$
\|u\|_{X}=\max \left\{\|u\|_{\infty},\left\|[\sigma(b)-\tau] u^{\Delta}\right\|_{\infty}\right\}, \quad \text { where } \tau(t):=t, \forall t \in \mathbb{T} .
$$

A function $x:[a, \sigma(b)] \rightarrow \mathbb{R}$ is said to be a solution of the problem (1.2) provided $x$ is $\Delta$ differentiable $\Delta$-a.e. on $[a, \sigma(b)), x^{\Delta}$ is $\nabla$-differentiable $\Delta \nabla$-a.e. on $(a, b], x^{\Delta \nabla}:(a, b] \rightarrow \mathbb{R}$ satisfies the dynamic equation in (1.2), and $x$ fulfills the boundary conditions in (1.2).

We make the following assumptions throughout this paper.

(A0) $\sigma(b) \neq \max \mathbb{T}, \xi_{i} \in(a, \sigma(b))$ for $i \in\{1,2, \ldots, m-2\}, a<\xi_{1}<\xi_{2}<\cdots<\xi_{m-2}<$ $\sigma(b), a_{i} \in \mathbb{R}$ for $i \in\{1,2, \ldots, m-2\}, m \geq 3$, and

$$
\sum_{i=1}^{m-2} a_{i} \neq 1 . \quad \text { We define } A:=1+\frac{\sum_{i=1}^{m-2}\left|a_{i}\right|}{\left|1-\sum_{i=1}^{m-2} a_{i}\right|}
$$

(A1) There exist $p, q, r \in E$ such that for $(u, v) \in \mathbb{R}^{2}$ we have

$$
|f(t, u, v)| \leq p(t)|u|+[\sigma(b)-t] q(t)|v|+r(t), \nabla \text {-a.e. on }(a, \sigma(b)]
$$

(A2) $e \in E$, that is, $e \in L_{\mathrm{loc}}^{\nabla}(a, \sigma(b))$ and $\int_{a}^{\sigma(b)}[\sigma(b)-\rho(t)]|e(t)| \nabla t<\infty$.

By (A1) and (A2), we allow $f(\cdot, u, v)$ and $e(\cdot)$ to be singular at $t=\sigma(b)$. When $\sigma(b)=b$, their singularities are clear. When $\sigma(b)>b$, their singularities are reflected on that both $f(\cdot, u, v)$ and $e(\cdot)$ may not be defined at $t=\sigma(b)$. If we put $f(\sigma(b), u, v)=\infty$, then

$$
\int_{a}^{\sigma(b)} f(t, u, v) \nabla t=\int_{a}^{b} f(t, u, v) \nabla t+f(\sigma(b), u, v)[\sigma(b)-b]=\infty .
$$

Now (2.16) means that $\infty=\infty$ provided $p(\sigma(b))=q(\sigma(b))=r(\sigma(b))=\infty$.

\section{Green's function and preliminary lemmas}

Let $G$ be Green's function of the second-order boundary value problem

$$
-x^{\Delta \nabla}=0, \quad \text { on }(a, b], \quad x^{\Delta}(a)=0, \quad x(\sigma(b))=0,
$$


6 Singular multipoint dynamic boundary value problems

which can be explicitly given by

$$
G(t, s)= \begin{cases}\sigma(b)-s & \text { if } a \leq t \leq s \leq \sigma(b) \\ \sigma(b)-t & \text { if } a \leq s \leq t \leq \sigma(b)\end{cases}
$$

From this explicit representation, the following lemma is clear.

LEMma 3.1. We have

$$
0 \leq G(t, s) \leq G(s, s), \quad \forall s, t \in[a, \sigma(b)] .
$$

For each $y \in E$, we define

$$
u(t)=\int_{a}^{\sigma(b)} G(t, s) y(s) \nabla s, \quad \text { for } t \in[a, \sigma(b)] .
$$

Since

$$
\begin{aligned}
\left|\int_{a}^{\sigma(b)} G(t, s) y(s) \nabla s\right| & \leq \int_{a}^{\sigma(b)} G(s, s)|y(s)| \nabla s \\
& =\int_{a}^{\sigma(b)}[\sigma(b)-s]|y(s)| \nabla s \\
& \leq \int_{a}^{\sigma(b)}[\sigma(b)-\rho(s)]|y(s)| \nabla s \\
& =\|y\|_{E}<\infty,
\end{aligned}
$$

we know that $u:[a, \sigma(b)] \rightarrow \mathbb{R}$ is well defined.

Lemma 3.2. Let $y \in E$. Then

$$
\int_{a}^{\sigma(b)} G(\cdot, s) y(s) \nabla s \in A C_{\operatorname{loc}}[a, \sigma(b)) .
$$

Proof. We have

$$
\int_{a}^{\sigma(b)} G(t, s) y(s) \nabla s=\int_{a}^{t}[\sigma(b)-t] y(s) \nabla s+\int_{t}^{\sigma(b)}[\sigma(b)-s] y(s) \nabla s .
$$

Since $y \in E$, we have $y \in L_{\mathrm{loc}}^{\nabla}(a, \sigma(b))$ and $[\sigma(b)-\tau] y \in L^{\nabla}(a, \sigma(b)]$. Thus (3.6) follows from Lemma 2.6.

Lemma 3.3. Let $y \in E$. Then

$$
\hat{y} \in L^{\nabla}(a, \sigma(b)], \quad \text { where } \hat{y}(t):=\int_{a}^{t} y(s) \nabla s, \forall t \in \mathbb{T} .
$$

Proof. Set

$$
\Phi(t, s)= \begin{cases}y(s) & \text { if } a \leq s \leq t \leq \sigma(b) \\ 0 & \text { if } a \leq t<s \leq \sigma(b)\end{cases}
$$


Since

$$
\begin{aligned}
\int_{a}^{\sigma(b)} \int_{a}^{\sigma(b)}|\Phi(t, s)| \nabla t \nabla s & =\int_{a}^{\sigma(b)} \int_{\rho(s)}^{\sigma(b)}|y(s)| \nabla t \nabla s \\
& =\int_{a}^{\sigma(b)}[\sigma(b)-\rho(s)]|y(s)| \nabla s=\|y\|_{E}<\infty,
\end{aligned}
$$

we get by the Fubini theorem [2] that

$$
\int_{a}^{t}|y(s)| \nabla s=\int_{a}^{\sigma(b)}|\Phi(t, s)| \nabla s \in L^{\nabla}(a, \sigma(b)] .
$$

Furthermore,

$$
\begin{aligned}
\int_{a}^{\sigma(b)}\left|\int_{a}^{t} y(s) \nabla s\right| \nabla t & =\int_{a}^{\sigma(b)}\left|\int_{a}^{\sigma(b)} \Phi(t, s) \nabla s\right| \nabla t \\
& \leq \int_{a}^{\sigma(b)} \int_{a}^{\sigma(b)}|\Phi(t, s)| \nabla s \nabla t \\
& =\int_{a}^{\sigma(b)} \int_{a}^{\sigma(b)}|\Phi(t, s)| \nabla t \nabla s<\infty .
\end{aligned}
$$

Thus (3.8) holds.

Lemma 3.4. Let $y \in E$. Then

$$
\lim _{t \rightarrow \sigma(b)} \int_{a}^{\sigma(b)} G(t, s) y(s) \nabla s=0 .
$$

Proof. We have

$$
\lim _{t \rightarrow \sigma(b)} \int_{a}^{\sigma(b)} G(t, s) y(s) \nabla s=\lim _{t \rightarrow \sigma(b)}\left\{\int_{a}^{t}[\sigma(b)-t] y(s) \nabla s+\int_{t}^{\sigma(b)}[\sigma(b)-s] y(s) \nabla s\right\} .
$$

Since $y \in E$, we have $[\sigma(b)-\tau] y \in L^{\nabla}(a, \sigma(b)]$. So

$$
\lim _{t \rightarrow \sigma(b)} \int_{t}^{\sigma(b)}[\sigma(b)-s] y(s) \nabla s=0
$$

Now we verify that

$$
\lim _{t \rightarrow \sigma(b)} \int_{a}^{t}[\sigma(b)-t] y(s) \nabla s=0
$$

holds, which completes the proof. We have

$$
\begin{aligned}
\int_{t}^{\sigma(b)} \int_{a}^{r} y(s) \nabla s \nabla r & =\left|r \int_{a}^{r} y(s) \nabla s\right|_{t}^{\sigma(b)}-\int_{t}^{\sigma(b)} \rho(r) y(r) \nabla r \\
& =[\sigma(b)-t] \int_{a}^{t} y(s) \nabla s+\int_{t}^{\sigma(b)}[\sigma(b)-\rho(s)] y(s) \nabla s .
\end{aligned}
$$


8 Singular multipoint dynamic boundary value problems

Since $y \in E$, we have $[\sigma(b)-\rho] y \in L^{\nabla}(a, \sigma(b)]$, so

$$
\lim _{t \rightarrow \sigma(b)} \int_{t}^{\sigma(b)}[\sigma(b)-\rho(s)] y(s) \nabla s=0
$$

On the other hand, we know from Lemma 3.3 that $\hat{y} \in L^{\nabla}(a, \sigma(b)]$, so

$$
\lim _{t \rightarrow \sigma(b)} \int_{t}^{\sigma(b)} \int_{a}^{r} y(s) \nabla s \nabla r=\lim _{t \rightarrow \sigma(b)} \int_{t}^{\sigma(b)} \hat{y}(r) \nabla r=0 .
$$

Therefore the limit in (3.16) exists and is equal to zero, that is, (3.16) holds.

For each $y \in E$, we define

$$
(T y)(t)=\int_{a}^{\sigma(b)} G(t, s) y(s) \nabla s+\frac{1}{1-\sum_{i=1}^{m-2} a_{i}} \sum_{i=1}^{m-2} a_{i} \int_{a}^{\sigma(b)} G\left(\xi_{i}, s\right) y(s) \nabla s .
$$

Now since (using Lemma 3.1 and the notation introduced in (A0))

$$
\begin{aligned}
|(T y)(t)| & \leq \int_{a}^{\sigma(b)} G(s, s)|y(s)| \nabla s+\frac{1}{\left|1-\sum_{i=1}^{m-2} a_{i}\right|} \sum_{i=1}^{m-2}\left|a_{i}\right| \int_{a}^{\sigma(b)} G(s, s)|y(s)| \nabla s \\
& =A \int_{a}^{\sigma(b)}[\sigma(b)-s]|y(s)| \nabla s \leq A\|y\|_{E}<\infty,
\end{aligned}
$$

we know from $(\mathrm{A} 0)$ that $T y:[a, \sigma(b)] \rightarrow \mathbb{R}$ is well defined.

Lemma 3.5. Let $y \in E$. Then $T y \in X$ and

$$
(T y)^{\Delta \nabla}+y=0, \quad \Delta \nabla \text {-a.e. on }(a, b] .
$$

Proof. By using Lemma 3.2, Ty $\in A C_{\text {loc }}[a, \sigma(b))$ for $y \in E$. Together with Lemma 2.9, we have that $T y$ is $\Delta$-differentiable $\Delta$-a.e. on $[a, \sigma(b))$. Then

$$
(T y)^{\Delta}(t)=-\int_{a}^{t} y(s) \nabla s
$$

so $(T y)^{\Delta} \in A C_{\mathrm{loc}}[a, \sigma(b))$ since $y \in L_{\mathrm{loc}}^{\nabla}(a, \sigma(b))$. Next,

$$
(T y)^{\Delta \nabla}(t)=-y(t), \quad \Delta \nabla \text {-a.e. on }(a, b] .
$$

(Note that $\mu_{\nabla}(\{b\})=b-\rho(b)>0$ when $\rho(b)<b$. If $\sigma(b)=b$ holds at the same time, that is, $b$ is an lsrd point, then this equality just holds for $\Delta \nabla$-a.e. $t \in(a, b)$. Further, by means of the definition of $\Delta \nabla$-a.e. and the fact of $\mu_{\Delta}(\{b\})=0$ for $\sigma(b)=b$, we get (3.24).) Next, since

$$
(T y)(t)=\int_{a}^{t}(T y)^{\Delta}(s) \Delta s+(T y)(a)
$$


and $(T y)^{\Delta} \in L^{\nabla}(a, \sigma(b)]$ from Lemma 3.3, we have $T y \in A C[a, \sigma(b)]$ by means of Lemma 2.6. Now we need to verify that $\lim _{t \rightarrow \sigma(b)}[\sigma(b)-t](T y)^{\Delta}(t)$ exists. Indeed, according to

$$
\lim _{t \rightarrow \sigma(b)}[\sigma(b)-t](T y)^{\Delta}(t)=-\lim _{t \rightarrow \sigma(b)} \int_{a}^{t}[\sigma(b)-t] y(s) \nabla s,
$$

we obtain the existence of the above limit from the proof of Lemma 3.4. Therefore, $T y \in$ $X$ when $y \in E$. The proof is complete.

Lemma 3.6. Let $y \in E$. Then

$$
(T y)^{\Delta}(a)=0, \quad(T y)(\sigma(b))=\sum_{i=1}^{m-2} a_{i}(T y)\left(\xi_{i}\right) .
$$

Proof. The fact that $(T y)^{\Delta} \in C[a, \sigma(b))$ and $y \in L_{\mathrm{loc}}^{\nabla}(a, \sigma(b))$ imply that

$$
(T y)^{\Delta}(a)=\lim _{t \rightarrow a}(T y)^{\Delta}(t)=-\lim _{t \rightarrow a} \int_{a}^{t} y(s) \nabla s=0 .
$$

From $T y \in C[a, \sigma(b)]$ and Lemma 3.4, we have

$$
\begin{aligned}
(T y)(\sigma(b)) & =\lim _{t \rightarrow \sigma(b)}(T y)(t) \\
& =\lim _{t \rightarrow \sigma(b)} \int_{a}^{\sigma(b)} G(t, s) y(s) \nabla s+\frac{1}{1-\sum_{i=1}^{m-2} a_{i}} \sum_{i=1}^{m-2} a_{i} \int_{a}^{\sigma(b)} G\left(\xi_{i}, s\right) y(s) \nabla s \\
& =\frac{1}{1-\sum_{i=1}^{m-2} a_{i}} \sum_{i=1}^{m-2} a_{i} \int_{a}^{\sigma(b)} G\left(\xi_{i}, s\right) y(s) \nabla s .
\end{aligned}
$$

By (3.20), we have

$$
\begin{aligned}
\sum_{i=1}^{m-2} a_{i}(T y)\left(\xi_{i}\right) & =\sum_{i=1}^{m-2} a_{i}\left[\int_{a}^{\sigma(b)} G\left(\xi_{i}, s\right) y(s) \nabla s+\frac{1}{1-\sum_{i=1}^{m-2} a_{i}} \sum_{i=1}^{m-2} a_{i} \int_{a}^{\sigma(b)} G\left(\xi_{i}, s\right) y(s) \nabla s\right] \\
& =\frac{1}{1-\sum_{i=1}^{m-2} a_{i}} \sum_{i=1}^{m-2} a_{i} \int_{a}^{\sigma(b)} G\left(\xi_{i}, s\right) y(s) \nabla s=(T y)(\sigma(b)) .
\end{aligned}
$$

This completes the proof.

For $x \in X$, we define a nonlinear operator $N$ by

$$
(N x)(t)=-f\left(t, x(t), x^{\Delta}(t)\right)-e(t), \quad \text { for } t \in(a, \sigma(b)) .
$$


10 Singular multipoint dynamic boundary value problems

From (A1) and (A2), we conclude that $N: X \rightarrow E$ is well defined. In fact, for $d<\sigma(b)$,

$$
\begin{aligned}
\int_{a}^{d}|(N x)(t)| \nabla t & \leq \int_{a}^{d}\left|f\left(t, x(t), x^{\Delta}(t)\right)\right| \nabla t+\int_{a}^{d}|e(t)| \nabla t \\
& \leq \int_{a}^{d} p(t)|x(t)| \nabla t+\int_{a}^{d}[\sigma(b)-t] q(t)\left|x^{\Delta}(t)\right| \nabla t+\int_{a}^{d} r(t) \nabla t+\int_{a}^{d}|e(t)| \nabla t \\
& \leq\|x\|_{X}\left(\int_{a}^{d} p(t) \nabla t+\int_{a}^{d} q(t) \nabla t\right)+\int_{a}^{d} r(t) \nabla t+\int_{a}^{d}|e(t)| \nabla t<\infty .
\end{aligned}
$$

So $N x \in L_{\mathrm{loc}}^{\nabla}(a, \sigma(b))$. Moreover,

$$
\begin{aligned}
&\left|\int_{a}^{\sigma(b)}[\sigma(b)-\rho(t)](N x)(t) \nabla t\right| \\
& \leq \int_{a}^{\sigma(b)}[\sigma(b)-\rho(t)]\left|f\left(t, x(t), x^{\Delta}(t)\right)+e(t)\right| \nabla t \\
& \leq \int_{a}^{\sigma(b)}[\sigma(b)-\rho(t)] p(t)|x(t)| \nabla t \\
&+\int_{a}^{\sigma(b)}[\sigma(b)-\rho(t)][\sigma(b)-t] q(t)\left|x^{\Delta}(t)\right| \nabla t \\
&+\int_{a}^{\sigma(b)}[\sigma(b)-\rho(t)] r(t) \nabla t+\int_{a}^{\sigma(b)}[\sigma(b)-\rho(t)]|e(t)| \nabla t \\
& \leq\|p\|_{E}\|x\|_{\infty}+\|q\|_{E}\left\|[\sigma(b)-\tau] x^{\Delta}\right\|_{\infty}+\|r\|_{E}+\|e\|_{E} \\
& \leq\|x\|_{X}\left(\|p\|_{E}+\|q\|_{E}\right)+\|r\|_{E}+\|e\|_{E}<\infty .
\end{aligned}
$$

Thus $[\sigma(b)-\rho](N x) \in L^{\nabla}(a, \sigma(b)]$.

LeMma 3.7. TN $: X \rightarrow X$ is completely continuous.

Proof. By the definitions of $T$ and $N$, we get that

$$
\begin{aligned}
((T N) x)(t)= & -\int_{a}^{\sigma(b)} G(t, s) f\left(s, x(s), x^{\Delta}(s)\right) \nabla s-\int_{a}^{\sigma(b)} G(t, s) e(s) \nabla s \\
& -\frac{1}{1-\sum_{i=1}^{m-2} a_{i}} \sum_{i=1}^{m-2} a_{i} \int_{a}^{\sigma(b)} G\left(\xi_{i}, s\right) f\left(s, x(s), x^{\Delta}(s)\right) \nabla s \\
& -\frac{1}{1-\sum_{i=1}^{m-2} a_{i}} \sum_{i=1}^{m-2} a_{i} \int_{a}^{\sigma(b)} G\left(\xi_{i}, s\right) e(s) \nabla s .
\end{aligned}
$$

For each $x_{1}, x_{2} \in X$,

$$
\begin{aligned}
& \left\|(T N) x_{1}-(T N) x_{2}\right\|_{X} \\
& \left.\quad=\max \left\{\left\|(T N) x_{1}-(T N) x_{2}\right\|_{\infty}, \|[\sigma(b)-\tau]\left[(T N) x_{1}\right)^{\Delta}-\left((T N) x_{2}\right)^{\Delta}\right] \|_{\infty}\right\} .
\end{aligned}
$$


Since $f$ satisfies Carathéodory's conditions, together with (use (A0) and Lemma 3.1)

$$
\begin{aligned}
& \left|(T N) x_{1}-(T N) x_{2}\right| \\
& \leq \int_{a}^{\sigma(b)} G(t, s)\left|f\left(s, x_{2}(s), x_{2}^{\Delta}(s)\right)-f\left(s, x_{1}(s), x_{1}^{\Delta}(s)\right)\right| \nabla s \\
& \quad+\frac{1}{\left|1-\sum_{i=1}^{m-2} a_{i}\right|} \sum_{i=1}^{m-2}\left|a_{i}\right| \int_{a}^{\sigma(b)} G\left(\xi_{i}, s\right)\left|f\left(s, x_{2}(s), x_{2}^{\Delta}(s)\right)-f\left(s, x_{1}(s), x_{1}^{\Delta}(s)\right)\right| \nabla s \\
& \quad \leq A \int_{a}^{\sigma(b)} G(s, s)\left|f\left(s, x_{2}(s), x_{2}^{\Delta}(s)\right)-f\left(s, x_{1}(s), x_{1}^{\Delta}(s)\right)\right| \nabla s, \\
& \left|[\sigma(b)-t]\left[\left((T N) x_{1}\right)^{\Delta}(t)-\left((T N) x_{2}\right)^{\Delta}(t)\right]\right| \\
& \leq[\sigma(b)-a] \int_{a}^{t}\left|f\left(s, x_{1}(s), x_{1}^{\Delta}(s)\right)-f\left(s, x_{2}(s), x_{2}^{\Delta}(s)\right)\right| \nabla s,
\end{aligned}
$$

it is easy to show that $T N: X \rightarrow X$ is continuous.

Now let $B \subset X$ be a bounded set. We need to show that $(T N)(B) \subset X$ is a relatively compact subset. Let $\left\{x_{n}\right\}_{n=1}^{\infty} \subset B$ and denote

$$
w_{n}(t)=\left((T N) x_{n}\right)(t), \quad z_{n}(t)=[\sigma(b)-t]\left((T N) x_{n}\right)^{\Delta}(t)
$$

We only need to show that there exists a subsequence with

$$
\begin{array}{cc}
w_{n} \longrightarrow w^{*}, & \text { in } C[a, \sigma(b)], \\
z_{n} \longrightarrow z^{*}, & \text { in } C[a, \sigma(b)],
\end{array}
$$

where $z^{*}(t)=[\sigma(b)-t]\left(w^{*}\right)^{\Delta}(t)$ for $t \in[a, \sigma(b))$. We prove (3.38) and (3.39) by the following three steps.

Step 1. We prove that $(T N)(B)$ is bounded. Let $M=\sup \left\{\|x\|_{X}: x \in B\right\}$. Then $M$ is a finite number. For each $t \in(a, \sigma(b))$, we have

$$
\begin{aligned}
\left|\left(N x_{n}\right)(t)\right| & \leq\left|f\left(t, x_{n}(t), x_{n}^{\Delta}(t)\right)\right|+|e(t)| \\
& \leq p(t)|| x_{n} \|_{\infty}+[\sigma(b)-t] q(t)\left|x_{n}^{\Delta}(t)\right|+r(t)+|e(t)| \\
& \leq p(t) M+q(t) M+r(t)+|e(t)|:=\chi(t) .
\end{aligned}
$$

Clearly, (A1) and (A2) imply that $\chi \in E$. Thus

$$
\left\|N x_{n}\right\|_{E} \leq \int_{a}^{\sigma(b)}[\sigma(b)-\rho(t)] \chi(t) \nabla t:=K<\infty .
$$

It follows that $T\left(\left(N x_{n}\right)(t)\right)$ is bounded. So $(T N)(B)$ is bounded. 
12 Singular multipoint dynamic boundary value problems

Step 2. We prove that $\left\{w_{n}\right\}_{n=1}^{\infty}$ is equicontinuous on $[a, \sigma(b)]$. For every $t_{1}, t_{2} \in[a, \sigma(b)]$ with $t_{1}<t_{2}$, according to Lemma 2.11(i), (3.23), and (3.40), we get

$$
\begin{aligned}
\left|w_{n}\left(t_{1}\right)-w_{n}\left(t_{2}\right)\right| & =\left|\int_{t_{2}}^{t_{1}}\left((T N) x_{n}\right)^{\Delta}(t) \Delta t\right| \leq \int_{t_{1}}^{t_{2}}\left|\left((T N) x_{n}\right)^{\Delta}(t)\right| \Delta t \\
& =\int_{t_{1}}^{t_{2}}\left|\int_{a}^{t} N x_{n}(s) \nabla s\right| \Delta t \leq \int_{t_{1}}^{t_{2}} \int_{a}^{t}\left|N x_{n}(s)\right| \nabla s \Delta t \\
& \leq \int_{t_{1}}^{t_{2}} \int_{a}^{t} \chi(s) \nabla s \Delta t=\int_{t_{1}}^{t_{2}} \int_{a}^{\rho(t)} \chi(s) \nabla s \nabla t .
\end{aligned}
$$

By the proof of Lemma 3.3, $\hat{\chi} \circ \rho \in L^{\nabla}(a, \sigma(b)]$. Thus (3.42) shows that $\left\{w_{n}\right\}_{n=1}^{\infty}$ is equicontinuous on $[a, \sigma(b)]$. Therefore by the Arzelà-Ascoli theorem, after taking a subsequence if necessary, (3.38) holds.

Step 3. We prove that $\left\{z_{n}\right\}_{n=1}^{\infty}$ is equicontinuous on $[a, \sigma(b)]$. For every $t_{1}, t_{2} \in[a, \sigma(b)]$ with $t_{1}<t_{2}$, we have by Lemma 3.5 and (3.40) that

$$
\begin{aligned}
\left|z_{n}\left(t_{1}\right)-z_{n}\left(t_{2}\right)\right| & \leq \int_{t_{1}}^{t_{2}}\left|z_{n}^{\nabla}(t)\right| \nabla t \\
& =\int_{t_{1}}^{t_{2}}\left|-\left((T N) x_{n}\right)^{\Delta}(t)+[\sigma(b)-\rho(t)]\left((T N) x_{n}\right)^{\Delta \nabla}(t)\right| \nabla t \\
& \leq \int_{t_{1}}^{t_{2}} \int_{a}^{t}\left|N x_{n}(s)\right| \nabla s \nabla t+\int_{t_{1}}^{t_{2}}[\sigma(b)-\rho(t)]\left|N x_{n}(t)\right| \nabla t \\
& \leq \int_{t_{1}}^{t_{2}} \int_{a}^{t} \chi(s) \nabla s \nabla t+\int_{t_{1}}^{t_{2}}[\sigma(b)-\rho(t)] \chi(t) \nabla t \\
& =\int_{t_{1}}^{t_{2}}\left[\int_{a}^{t} \chi(s) \nabla s+[\sigma(b)-\rho(t)] \chi(t)\right] \nabla t .
\end{aligned}
$$

Since $\chi \in E$ and because of Lemma 3.3, we know that

$$
\hat{\chi}+[\sigma(b)-\rho] \chi \in L^{\nabla}(a, \sigma(b)]
$$

Thus (3.43) shows that $\left\{z_{n}\right\}_{n=1}^{\infty}$ is equicontinuous on $[a, \sigma(b)]$. Therefore by the ArzelàAscoli theorem, after taking a subsequence if necessary, (3.39) holds.

This completes the proof.

\section{Main result}

Theorem 4.1. Let $f:(a, \sigma(b)) \times \mathbb{R}^{2} \rightarrow \mathbb{R}$ satisfy Carathéodory's conditions. Assume that (A0), (A1), and (A2) hold. Then the problem (1.2) has at least one solution in $X$ provided

$$
A\|p\|_{E}+\|q\|_{E}<1
$$

Proof. From Lemmas 3.5 and 3.6, we know that $x \in X$ is a solution of (1.2) if and only if

$$
x=T N x .
$$


By Lemma 3.7, we will apply the Leray-Schauder continuation theorem [17, Corollary IV.7] to obtain the existence of a solution for (4.2) in $X$. To do this, it suffices to verify that the set of all possible solutions of the family of equations

$$
\begin{aligned}
x^{\Delta \nabla} & =\lambda f\left(t, x, x^{\Delta}\right)+\lambda e(t) \quad \text { on }(a, b] \\
x^{\Delta}(a) & =0, \quad x(\sigma(b))=\sum_{i=1}^{m-2} a_{i} x\left(\xi_{i}\right)
\end{aligned}
$$

is, a priori, bounded in $X$ by a constant independent of $\lambda \in[0,1]$.

Let $x \in X$ be a solution of (4.3) for some $\lambda \in[0,1]$. Then for $t \in[a, \sigma(b)]$, we have

$$
\begin{aligned}
|x(t)| & =\left|\int_{a}^{\sigma(b)} G(t, s) \lambda(N x)(s) \nabla s+\frac{1}{1-\sum_{i=1}^{m-2} a_{i}} \sum_{i=1}^{m-2} a_{i} \int_{a}^{\sigma(b)} G\left(\xi_{i}, s\right) \lambda(N x)(s) \nabla s\right| \\
& \leq A \int_{a}^{\sigma(b)} G(s, s) \lambda|(N x)(s)| \nabla s \\
& =A \int_{a}^{\sigma(b)}[\sigma(b)-s]\left|x^{\Delta \nabla}(s)\right| \nabla s \\
& \leq A\left\|x^{\Delta \nabla}\right\|_{E} .
\end{aligned}
$$

This implies

$$
\|x\|_{\infty} \leq A\left\|x^{\Delta \nabla}\right\|_{E}
$$

Similarly,

$$
\begin{aligned}
\left|[\sigma(b)-t] x^{\Delta}(t)\right| & =\left|[\sigma(b)-t]\left[-\int_{a}^{t} \lambda(N x)(s) \nabla s\right]\right| \\
& \leq \int_{a}^{\sigma(b)}[\sigma(b)-\rho(s)]\left|x^{\Delta \nabla}(s)\right| \nabla s=\left\|x^{\Delta \nabla}\right\|_{E},
\end{aligned}
$$

and therefore

$$
\left\|[\sigma(b)-\tau] x^{\Delta}\right\|_{\infty} \leq\left\|x^{\Delta \nabla}\right\|_{E} .
$$

Now we get from (4.3), (4.5), (4.7), (A1), and (A2) that

$$
\begin{aligned}
\left\|x^{\Delta \nabla}\right\|_{E} & =\int_{a}^{\sigma(b)}[\sigma(b)-\rho(t)]\left|x^{\Delta \nabla}(t)\right| \nabla t \\
& =\int_{a}^{\sigma(b)} \lambda[\sigma(b)-\rho(t)]\left|f\left(t, x(t), x^{\Delta}(t)\right)+e(t)\right| \nabla t \\
& \leq \int_{a}^{\sigma(b)}[\sigma(b)-\rho(t)]\left[p(t)|x(t)|+[\sigma(b)-t] q(t)\left|x^{\Delta}(t)\right|+r(t)+|e(t)|\right] \nabla t \\
& \leq\|p\|_{E}\|x\|_{\infty}+\|q\|_{E}\left\|[\sigma(b)-\tau] x^{\Delta}\right\|_{\infty}+\|r\|_{E}+\|e\|_{E} \\
& \leq A\|p\|_{E}\left\|x^{\Delta \nabla}\right\|_{E}+\|q\|_{E}\left\|x^{\Delta \nabla}\right\|_{E}+\|r\|_{E}+\|e\|_{E} .
\end{aligned}
$$


Thus we get from the assumption (4.1) that

$$
\left\|x^{\Delta \nabla}\right\|_{E} \leq \frac{\|r\|_{E}+\|e\|_{E}}{1-\left(A\|p\|_{E}+\|q\|_{E}\right)}:=c .
$$

Here $c$ is a constant, which is independent of $\lambda \in[0,1]$. Therefore, by (4.5) and (4.7), we get

$$
\|x\|_{X}=\max \left\{\|x\|_{\infty},\left\|[\sigma(b)-\tau] x^{\Delta}\right\|_{\infty}\right\} \leq \max \{c, A c\}=A c .
$$

Therefore the proof is complete.

\section{References}

[1] R. P. Agarwal and D. O'Regan, Some new results for singular problems with sign changing nonlinearities, Journal of Computational and Applied Mathematics 113 (2000), no. 1-2, 1-15.

[2] C. D. Ahlbrandt and C. Morian, Partial differential equations on time scales, Journal of Computational and Applied Mathematics 141 (2002), no. 1-2, 35-55, Special issue on Dynamic Equations on Time Scales, edited by R. P. Agarwal, M. Bohner, and D. O'Regan.

[3] D. R. Anderson and R. I. Avery, An even-order three-point boundary value problem on time scales, Journal of Mathematical Analysis and Applications 291 (2004), no. 2, 514-525.

[4] H. Asakawa, Nonresonant singular two-point boundary value problems, Nonlinear Analysis Series A: Theory and Methods 44 (2001), no. 6, 791-809.

[5] F. M. Atici and G. Sh. Guseinov, On Green's functions and positive solutions for boundary value problems on time scales, Journal of Computational and Applied Mathematics 141 (2002), no. 1-2, 75-99, Special issue on Dynamic Equations on Time Scales, edited by R. P. Agarwal, M. Bohner, and D. O'Regan.

[6] M. Bohner and A. Peterson, Dynamic Equations on Time Scales. An Introduction with Applications, Birkhäuser Boston, Massachusetts, 2001.

[7] M. Bohner and A. Peterson (eds.), Advances in Dynamic Equations on Time Scales, Birkhäuser Boston, Massachusetts, 2003.

[8] J. J. DaCunha, J. M. Davis, and P. K. Singh, Existence results for singular three point boundary value problems on time scales, Journal of Mathematical Analysis and Applications 295 (2004), no. 2, 378-391.

[9] C. P. Gupta, S. K. Ntouyas, and P. Ch. Tsamatos, Solvability of an m-point boundary value problem for second order ordinary differential equations, Journal of Mathematical Analysis and Applications 189 (1995), no. 2, 575-584.

[10] Z. He, Existence of two solutions of m-point boundary value problem for second order dynamic equations on time scales, Journal of Mathematical Analysis and Applications 296 (2004), no. 1, 97-109.

[11] J. Henderson and E. R. Kaufmann, Focal boundary value problems for singular difference equations, Computers \& Mathematics with Applications 36 (1998), no. 10-12, 1-10.

[12] J. Henderson and W. Yin, Focal boundary-value problems for singular ordinary differential equations, Advances in Nonlinear Dynamics, Stability Control Theory Methods Appl., vol. 5, Gordon and Breach, Amsterdam, 1997, pp. 283-295.

[13] S. Hilger, Analysis on measure chains-a unified approach to continuous and discrete calculus, Results in Mathematics 18 (1990), no. 1-2, 18-56.

[14] R. Ma, Existence of positive solutions for superlinear semipositone m-point boundary-value problems, Proceedings of the Edinburgh Mathematical Society. Series II 46 (2003), no. 2, 279-292.

[15] R. Ma and H. Luo, Existence of solutions for a two-point boundary value problem on time scales, Applied Mathematics and Computation 150 (2004), no. 1, 139-147. 
[16] R. Ma and D. O'Regan, Solvability of singular second order m-point boundary value problems, Journal of Mathematical Analysis and Applications 301 (2005), no. 1, 124-134.

[17] J. Mawhin, Topological Degree Methods in Nonlinear Boundary Value Problems, CBMS Regional Conference Series in Mathematics, vol. 40, American Mathematical Society, Rhode Island, 1979.

[18] D. O’Regan, Theory of Singular Boundary Value Problems, World Scientific, New Jersey, 1994.

[19] S. D. Taliaferro, A nonlinear singular boundary value problem, Nonlinear Analysis 3 (1979), no. 6, 897-904.

[20] P. J. Y. Wong and R. P. Agarwal, On the existence of solutions of singular boundary value problems for higher order difference equations, Nonlinear Analysis 28 (1997), no. 2, 277-287.

[21] Y. Zhang, Positive solutions of singular sublinear Emden-Fowler boundary value problems, Journal of Mathematical Analysis and Applications 185 (1994), no. 1, 215-222.

[22] Z. Zhang and J. Wang, The upper and lower solution method for a class of singular nonlinear second order three-point boundary value problems, Journal of Computational and Applied Mathematics 147 (2002), no. 1, 41-52.

Martin Bohner: Department of Mathematics, University of Missouri-Rolla, Rolla,

MO 65409-0020, USA

E-mail address: bohner@umr.edu

Hua Luo: Department of Mathematics, Northwest Normal University, Lanzhou 730070,

Gansu, China

E-mail address: luohua@nwnu.edu.cn 